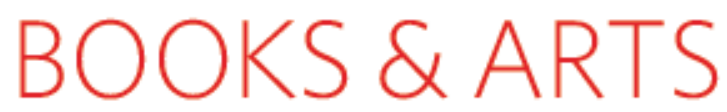

\title{
Physics ain't what it used to be
}

\section{Science is venturing into areas where experimental verification simply isn't possible.}

\author{
The Cosmic Landscape:String Theory and \\ the Illusion of Intel ligent Design \\ by Leonard Susskind \\ Little Brown: 2005. 416pp. \$24.95
}

\section{George Ellis}

Once upon a time, physics dealt with tangible objects - if you couldn'tweigh them or smash them together, at least you could observe them. As times changed, physicists started to deal with more ethereal things: electromagnetic fields and space-time metrics, for example. You couldn't see them but you could measure their influence on particle trajectories and so justifiably claim evidence of their existence. Nowadays things have changed. A phalanx of heavyweight physicists and cosmologists are claiming to prove the existence of other expanding universe domains even though there is no chance of observing them, nor any possibility of testing their supposed nature except in the most tenuous, indirect way.

How can this be a scientific proposal, when the core of science is testing theories against the evidence? In The Cosmic Landscape, Leonard Susskind argues that we should accept the reality of such universe domains on the basis of two theoretical elements that, taken together, could provide a solution to two major scientific conundrums. The first puzzle is the anthropic issue: the "apparent miracles of physics and cosmology" that make our existence possible. Many aspects of both physics and cosmology seem to be fine-tuned in such a way as to allow chemistry to function, planets to exist, and life to come into being. If they were substantially different, no life at all, and so no processes of darwinian evolution, would have occurred. Which particular aspect of this fine-tuning seems the most significant depends on one's discipline.

Susskind, a particle physicist, thinks the most important is the issue of the cosmological constant, relating to a universal repulsive force that acts on all matter. But this leads to the second conundrum: simple estimates suggest that this constant should be 120 orders of magnitude larger than recently observed. This is a major crisis for quantum field theory, which underlies these estimates. The link to the anthropic question is that if the constant were only twice as large, there would be no galaxies, stars, planets or life. The observed very small value of this constant, although contrary to

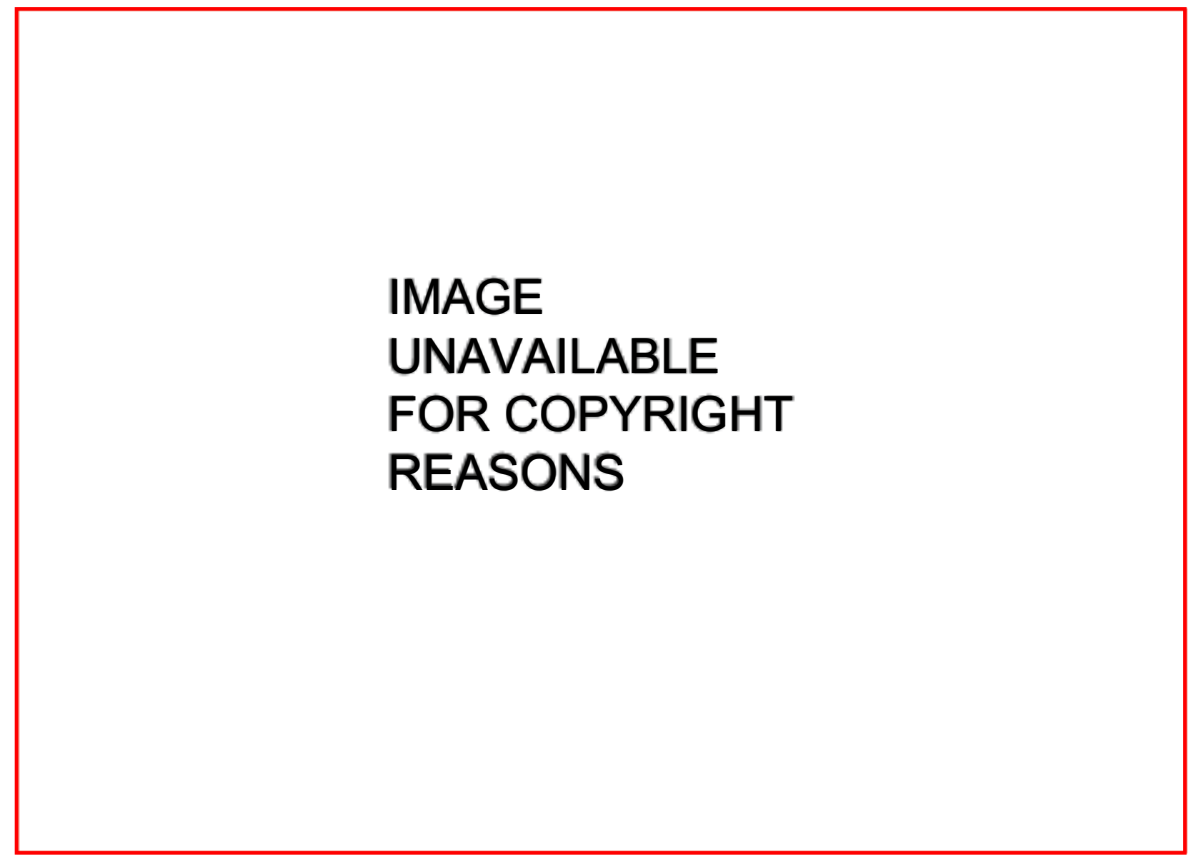

You gotta have faith: the idea of countless 'pocket universes' cannot be tested, so is it science?

our present theory of the quantum vacuum, is a necessary condition for our existence.

The first part of the proposed solution is the idea of a 'multiverse' - the existence of a huge number of 'pocket universes', like the vast expanding Universe domain we see around us, that are part of a much larger physical existence. These are supposed to arise through inflation, a process of extremely short-lived, very rapidly accelerating expansion that preceded the hot Big Bang era in the early Universe. 'Chaotic inflation' occurs if inflation is still occurring in distant domains around us today, forming overall a fractal-like structure of inflating domains and pocket universes.

The second part of the solution is the landscape of possibilities, a recent discovery in string theory, which is itself a proposed theory of fundamental physics that unites gravity with quantum physics. It has been suggested that the 'vacuum' of string theory is a structure of immensely complex possibilities, with each possible vacuum resulting in a different kind of local physics, for example, all possible values of the cosmological constant will occur in the different vacua of string theory. If we suppose that the pocket universes of chaotic inflation correspond to different vacua, then all possible kinds of local physics occur at different loca- tions somewhere in the multiverse. If enough combinations of possibilities are realized in this way, then the incredibly special conditions for life to exist will inevitably occur somewhere in the multiverse. The apparent design of conditions favourable to life in our own universe domain can therefore be explained in a naturalistic way.

This is an intriguing picture that unites quite disparate elements of physics and cosmology in a synthesis that is satisfying in many ways. But the question here is whether it is a scientific proposal, as there is no chance whatsoever of observationally verifying its main prediction, the existence of numerous other expanding universe domains beyond our visual horizon. We might hope to base our prediction that the multiverse exists on the fact it is an inevitable outcome of well established physics, but the physics underlying the proposal is hypothetical, rather than established. String theory is neither well defined nor experimentally proven, despite the energy and enthusiasm of its proponents, and there are alternative theories. The inflation field has not been uniquely identified in physical terms, much less shown to have the properties supposed in chaotic inflation.

We might hope to detect the multiverse 
indirectly by observing the remnants of the physical processes that underlie its existence; for example, the low value of the cosmological constant today could be such a hint. The problem here is that a multiverse proposal cannot in general be disproved this way, because if all possibilities exist somewhere in the multiverse, as some claim, then it can explain any observations, whatever they are. For example, no observations of anisotropy in the cosmic background radiation can disprove the multiverse hypothesis because all possible anisotropies will be generated in the different expanding universe domains; you just have to live in the right one.

The particular multiverse version proposed by Susskind, however, has the great virtue of being testable in one respect. It is supposed to have started out by quantum tunnelling, resulting in a spatially homogenous and isotropic universe with negative spatial curvature, and hence with a total density parameter $\Omega_{0}<1$ The best observationally determined value for this parameter, taking all the data into account, is $\Omega_{0}=1.02 \pm 0.02$. Taken at face value, this seems to contradict the proposed theory. But given the statistical uncertainties, the observations do not definitively exclude $\Omega_{0}<1$, so the theory survives; nevertheless, the observed value should be taken seriously in this era of 'precision cosmology'. These data are not discussed in the book - a symptom of some present-day cosmology, where faith in theory tends to trump evidence. Presumably the hope is that this observational result will go away as more evidence is collected.

The Cosmic Landscape is extremely well written, provides an excellent non-technical overview of the relevant physics, and tackles important questions in a lively way. However, it confuses the event horizon in the expanding universe with particle and visual horizons. In addition, like many multiverse writings, it uses the concept of infinity with gay abandon, when there is good reason - as pointed out by mathematician David Hilbert - to claim that it is not a good physical concept. The book also tries to justify the multiverse idea in terms of the 'manyworlds' interpretation of quantum theory - an unproven and totally profligate viewpoint that many find difficult to take seriously.

As a philosophical proposal, the multiverse idea is interesting and has considerable merit. The challenge facing cosmologists now is how to put on a sound basis the attempts to push science beyond the boundary where verification is possible - and what label to attach to the resultant theories. Physicists indulging in this kind of speculation sometimes denigrate philosophers of science, but they themselves do not yet have rigorous criteria to offer for proof of physical existence. This is what is needed to make this area solid science, rather than speculation. Until then, the multiverse situation seems to fit St Paul's description: "Faith is the substance of things hoped for, the evidence of things not seen." In this case, it is faith that enormous extrapolations from tested physics are correct; hope that correct hints as to the way things really are have been identified from all the possibilities, and that the present marginal evidence to the contrary will go

\section{Pet project}

The Dog and Its Genome

edited by Elaine A. Ostrander, Urs Giger

\& Kerstin Lindblad-Toh

Cold Spring Harbor Laboratory Press: 2005.

584 pp. $\$ 135, £ 80$

\section{Stephen J. O'Brien}

Genome technology has found its way into the living room with the completion of the wholegenome sequence of the domestic dog Canis familiaris, from a female boxer called Tasha. Finished just a year after its initiation in 2003, the remarkably complete sequence (representing an estimated $99 \%$ of the dog's 2.4 billion base pairs) achieves 7.5 -fold coverage of the genome and is a major advance over the 1.5fold sequence of a poodle published by Celera in 2003. The dog is now a front-line model for the discovery of disease genes, for gene annotation, and for probing the evolutionary roots of our mammalian origins. The Dog and Its Genome, edited by Elaine Ostrander, Urs Giger and Kerstin Lindblad-Toh, celebrates the completion of the dog sequence with 26 chapters on the genomic biology of man's best friend.

The book should appeal to dog fanciers, to genome biologists who wonder about the sequence's applications, and to students of comparative genomics. It presents well written and concise discussions of the history of dog breeds - there are generally estimated to be between 350 and 1,000, of which the American Kennel Club recognizes about 150 that do not exchange genes. As many as 20 breeds were developed by 1750 , increasing to 76 by 1905. Yet the domestication of dogs can be traced back 14,000 years on the evidence of archaeological remains, maybe even 40,000 years based on molecular comparisons with wolves. Clearly, dogs are the oldest domesticated species, as detailed in two of the book's chapters, and the phylogenetic ancestry of dog breeds is described in three chapters.

Years from now, as dog genomics matures, this volume will be remembered as the starting point, with vivid pieces on the vast phenotypic variation described for dogs. The latest interpretation of dog genome status is presented for experts and aficionados alike. The remarkable history of inbreeding has led to a mosaic genome of alternating homozygous and heterozygous/polymorphic segments specific for each breed; these are particularly useful for linkage disequilibrium-based association mapping of complex or multifactorial traits. away. This book gives a great overview of this important terrain, as seen from an enthusiast's viewpoint.

George Ellis is in the Department of Mathernatics, University of Cape Town, Cape Town 7701, South Africa.

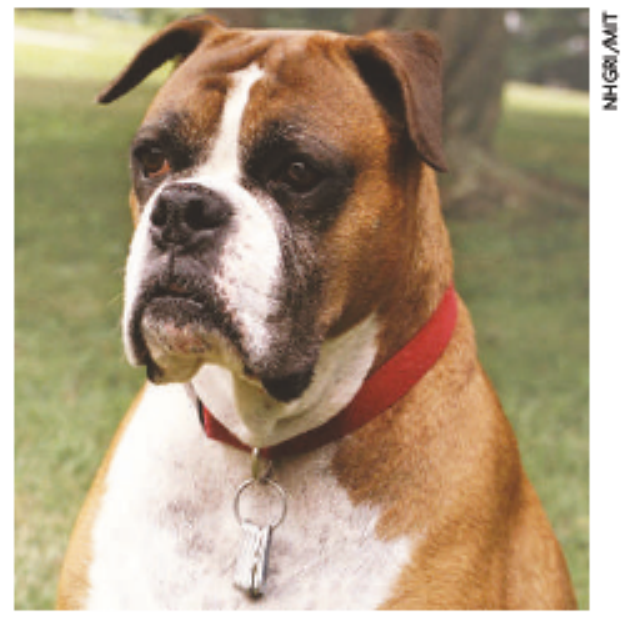

Boxer tricks: Tasha's genome will helpresearchers tounderstand human genetic diseases.

And dogs certainly have complex traits, notably the vast morphologic variation found in dog breeds as disparate as the chihuahua and the great dane. Dogs also have hard-wired behavioural acumen that allow them to herd livestock, locate missing persons and even sniff out human cancers at early stages. And of course they are loving companions like no other animals.

Generations of veterinary clinicians have identified nearly 500 human hereditary disease homologues in dogs, nearly all breed-specific; the 50 reviewed here have a confirmed genetic basis. Several have been treated successfully with futuristic gene-therapy protocols that should whet the appetite of the medical community. The book describes a cancer registry that documents the incidence and pathologies of a dozen neoplasms that account for $23 \%$ of deaths in the 65 million pet dogs in the United States. The challenge now will be to use the genome to detail the genetic bases of behaviours, morphological breed distinctiveness and the disposition of breed-specific cancers.

Researchers already have 'bibles' that define gene-based phenotypes suitable for interrogation by mouse, rat, fruitfly and human genetics. The Dog and Its Genome does the same for the canine genomics community. It should be consumed by researchers and their students quickly before forthooming advances render it dated on their bookshelves.

Stephen J. O'Brien is in the Laboratory of Genomic Diversity, National Cancer Institute, Frederick, Maryland 21702-1201, USA. 